\title{
RINGS SATISFYING MONOMIAL IDENTITIES
}

\author{
MOHAN S. PUTCHA AND ADIL YAQUB
}

\begin{abstract}
The following theorem is proved: Suppose $R$ is an associative ring and suppose that $w\left(x_{1}, \cdots, x_{n}\right)$ is a fixed word distinct from $x_{1} \cdots x_{n}$. If, further, $x_{1} \cdots x_{n}=w\left(x_{1}, \cdots, x_{n}\right)$, for all $x_{1}, \cdots, x_{n}$ in $R$, then the commutator ideal of $R$ is nilpotent. Moreover, it is shown that this theorem need not be true if the word $w$ is not fixed.
\end{abstract}

Suppose $R$ is an associative ring and suppose $x_{1}, \cdots, x_{n}$ are elements of $R$. A word $w\left(x_{1}, \cdots, x_{n}\right)$ in $x_{1}, \cdots, x_{n}$ is a product in which each factor is $x_{i}$ for some $i=1, \cdots, n$. Our present object is to prove

THEOREM 1. Suppose $R$ is an associative ring and suppose $w\left(x_{1}, \cdots, x_{n}\right)$ is a fixed word distinct from the word $x_{1} \cdots x_{n}$. Suppose

$$
x_{1} \cdots x_{n}=w\left(x_{1}, \cdots, x_{n}\right), \text { for all } x_{1}, \cdots, x_{n} \text { in } R \text {. }
$$

Then there exists a positive integer $m$ such that $R^{m} C(R) R^{m}=(0)$, where $C(R)$ is the commutator ideal of $R$. In particular, the commutator ideal of $R$ is nilpotent.

Moreover, a counterexample is given which shows that Theorem 1 need not be true if $w\left(x_{1}, \cdots, x_{n}\right)$ is not a fixed word.

In preparation for the proof of Theorem 1, we first show the following lemmas.

LEMMA 1. Suppose $R$ is an associative semisimple ring, and suppose $w\left(x_{1}, \cdots, x_{n}\right)$ is a fixed word involving each of the elements $x_{1}, \cdots, x_{n}$ of R. If, further,

$$
x_{1} \cdots x_{n}=w\left(x_{1}, \cdots, x_{n}\right), \text { for all } x_{1}, \cdots, x_{n} \text { in } R,
$$

then $R$ is commutative.

Proof. Suppose, first, that $R$ has an identity 1 . We now distinguish two cases.

Case 1.

$$
x_{1} \cdots x_{n}=w\left(x_{1}, \cdots, x_{n}\right)=x_{\sigma(1)} \cdots x_{\sigma(n)},
$$

Received by the editors February 23, 1971 and, in revised form, May 18, 1971. AMS 1970 subject classifications. Primary 16A38, 16A48; Secondary 16A70.

(c) American Mathematical Society 1972 
where $\sigma$ is a permutation of $\{1, \cdots, n\}$ distinct from the identity permutation. Then, for some integers $i, j$, we have $i<j$ but $\sigma(i)>\sigma(j)$. Now, let $a, b \in R$, and set in (3), $x_{\sigma(i)}=a, x_{\sigma(j)}=b, x_{k}=1$ for all $k \neq \sigma(i), k \neq \sigma(j)$, we get $b a=a b$, and the lemma follows.

Case 2.

$$
x_{1} \cdots x_{n}=w\left(x_{1}, \cdots, x_{n}\right)
$$

some $x_{t}$ appears at least twice in $w\left(x_{1}, \cdots, x_{n}\right)$.

In this case, by setting $x_{1}=\cdots=x_{t-1}=x_{t+1}=\cdots=x_{n}=1$ in (4), we get

$$
x_{t}=x_{t}^{k}, \text { for all } x_{t} \text { in } R \quad(k>1) .
$$

Hence [2, p. 217], $R$ is commutative, and the lemma follows again.

Returning to the general case, observe that, since $R$ is semisimple, $R$ is isomorphic to a subdirect sum of primitive rings $R_{i}, i \in \Gamma$, each of which clearly satisfies (2). Since every subring and every homomorphic image of $R$ satisfies (2), it follows [2, p. 33] that some complete matrix ring, $\Delta_{m}$, over a division ring satisfies (2) also. Since $\Delta_{m}$ has an identity, it follows (by the first part of this proof) that $\Delta_{m}$ is commutative. Thus $m=1$, and $\Delta_{m}=\Delta$ is a field. Hence [2, p. 33] the primitive ring $R_{i}$ is isomorphic to the field $\Delta$. Thus $R$ is isomorphic to a subdirect sum of fields, and hence $R$ is commutative. This proves the lemma.

Next, we consider the case in which the word $w\left(x_{1}, \cdots, x_{n}\right)$ satisfies (4). In this case, we can even say more. Indeed, we have

Lemma 2. Suppose $R$ is an associative ring and suppose that $C(R)$ and $J$ denote the commutator ideal and Jacobson ideal of $R$. Suppose that $w\left(x_{1}, \cdots, x_{n}\right)$ is a fixed word involving each of the elements $x_{1}, \cdots, x_{n}$ of $R$. Suppose, moreover, that for some $t, x_{t}$ appears at least twice in $w\left(x_{1}, \cdots, x_{n}\right)$. If, further,

$$
x_{1} \cdots x_{n}=w\left(x_{1}, \cdots, x_{n}\right), \text { for all } x_{1}, \cdots, x_{n} \text { in } R,
$$

then (i) $R / J$ is isomorphic to a subdirect sum of finite fields of orders bounded by the length of $w$; (ii) $C(R) \subseteq J$; (iii) $J$ consists of precisely the set of nilpotent elements of $R$.

Proof. Since $R / J$ is a semisimple ring which, clearly, satisfies (6), it follows, by Lemma 1 , that $R / J$ is commutative, and hence $R / J$ is isomorphic to a subdirect sum of fields $F_{i}, i \in \Gamma$. Now, each $F_{i}$ clearly satisfies (6), and hence by setting $x_{i}=1$ for all $i \neq t$ in (6), we obtain

$$
x_{t}=x_{t}^{k}, \text { for all } x_{t} \text { in } R \quad(k>1) .
$$

Therefore $F_{i}$ is a finite field with at most $k$ elements, and, clearly, $k$ is equal to or less than the length of the word $w\left(x_{1}, \cdots, x_{n}\right)$. This proves (i). 
Part (ii) follows at once, since $R / J$ is commutative. Finally, to prove (iii), suppse $a \in J$, and set $x_{i}=a$, for all $i$, in (6). We get, $a^{n}=a^{n} a^{l}$ for some $l \geqq 1$, and hence $a^{n}=0$. Conversely, if $a$ is nilpotent, then $\bar{a}(=a+J)$ is a nilpotent element in $R / J$, and hence by (i), $\bar{a}=\overline{0}$. Thus $a \in J$, and the lemma is proved.

Next, we prove

LEMMA 3. Suppose $R$ is an associative ring, and suppose $J$ is the Jacobson radical of $R$. Suppose that $w\left(x_{1}, \cdots, x_{n}\right)$ is a fixed word involving each of the elements $x_{1}, \cdots, x_{n}$ of $R$ and in which some $x_{t}$ appears at least twice. Suppose, moreover, that

$$
x_{1} \cdots x_{n}=w\left(x_{1}, \cdots, x_{n}\right), \text { for all } x_{1}, \cdots, x_{n} \text { in } R .
$$

Then, for some $i, 1 \leqq i \leqq n$, we have $R^{i-1} J R^{n-i}=(0)$.

Proof. By Lemma 2 (iii), $J$ is a nil ring. Now, let $a \in J$, and set in (8), $x_{1}=\cdot \cdot=x_{n}=a$, we get $a^{n}=a^{n} a^{l}$, for some $l \geqq 1$. Therefore the nil ring $J$ satisfies $a^{n}=0$, and thus $\left[1\right.$, p. 28] $J$ is locally nilpotent. Next, let $a_{1}, \cdots$, $a_{n} \in J$. Then the ring generated by $a_{1}, \cdots, a_{n}$ is nilpotent, say of index $k$. Now, by reiterating (8) until the length of the word $w\left(x_{1}, \cdots, x_{n}\right)$ in the right-hand side becomes $\geqq k$, it follows that $a_{1} \cdots a_{n}=0$, and hence $J^{n}=$ (0). Next, since $x_{i}$ appears at least twice in the word $w\left(x_{1}, \cdots, x_{n}\right)$, we can, by reiterating in (8), obtain a word $w^{\prime}\left(x_{1}, \cdots, x_{n}\right)$ of length $\geqq n^{2}$ and such that

$$
x_{1} \cdots x_{n}=w^{\prime}\left(x_{1}, \cdots, x_{n}\right), \text { for all } x_{1}, \cdots, x_{n} \text { in } R .
$$

Observe that in the word $w^{\prime}\left(x_{1}, \cdots, x_{n}\right)$, some $x_{i}$ appears at least $n$ times. We now fix $i$, and substitute $x_{i}=a ; x_{j}=r_{j}, j \neq i$, where each $r_{j} \in R$, we get

$$
r_{1} \cdots r_{i-1} a r_{i+1} \cdots r_{n} \in J^{n}=(0) \text {. }
$$

Hence, $R^{i-1} J R^{n-i}=(0)$, and the lemma is proved.

Our final lemma is true for semigroups (and hence, a fortiori, for rings), and has been proved in [4, Theorem 1].

LEMMA 4. Let $S$ be a semigroup such that, for all $x_{1}, \cdots, x_{n}$ in $S$,

$$
x_{1} \cdots x_{n}=x_{\sigma(1)} \cdots x_{\sigma(n)},
$$

where $\sigma$ is a fixed permutation of $\{1, \cdots, n\}$ distinct from the identity permutation. Then there exists an integer $m$ such that, for all $u, v$ in $S^{m}$ and all $x, y$ in $S$, we have uxyv=uyxv.

We are now in a position to prove Theorem 1. 
Proof of Theorem 1. First, suppose the word $w\left(x_{1}, \cdots, x_{n}\right)$ does not involve $x_{i}$, for some $i$. In (1), set $x_{i}=0$ and, for $j \neq i$, let $x_{j}$ be arbitrary; we get $w\left(x_{1}, \cdots, x_{n}\right) \equiv 0$ and hence, by (1), $x_{1} \cdots x_{n}=0$ for all $x_{1}, \cdots, x_{n}$ in $R$ (since $w\left(x_{1}, \cdots, x_{n}\right)$ is fixed). Thus $R^{n}=(0)$, and Theorem 1 follows at once. Next, suppose $w\left(x_{1}, \cdots, x_{n}\right)=x_{\sigma(1)} \cdots x_{\sigma(n)}$, for some permutation $\sigma$ of $\{1, \cdots, n\}$ different from the identity. Then, by Lemma 4,

$$
u(x y-y x) v=0, \text { for all } u, v \in R^{m} \text { and all } x, y \in R \text {. }
$$

Hence, $R^{m} C(R) R^{m}=(0)$, and Theorem 1 follows again. The only case left is when $w\left(x_{1}, \cdots, x_{n}\right)$ involves each $x_{i}$ and, moreover, some $x_{t}$ appears at least twice in $w\left(x_{1}, \cdots, x_{n}\right)$. By Lemmas 2 and 3 we have $R^{i-1} C(R) R^{n-i} \subseteq$ $R^{i-1} J R^{n-i}=(0)$, for some $i, 1 \leqq i \leqq n$, and once again the theorem follows. This completes the proof.

COROLlaRY. Suppose $R$ is an associative semiprime ring satisfying the hypothesis of Theorem 1. Then $R$ is commutative.

Proof. Since $R$ is a semiprime ring, the prime radical of $R$ is (0) [3, p. 146], and hence $R$ contains no nonzero nilpotent ideals. Now, by Theorem 1 , the commutator ideal, $C(R)$, of $R$ is nilpotent, and hence $C(R)=(0)$. Therefore $R$ is commutative, and the corollary is proved.

We conclude with the following

REMARK. Theorem 1 need not be true if we replace the fixed word $w\left(x_{1}, \cdots, x_{n}\right)$ by a "variable" word (depending on $\left.x_{1}, \cdots, x_{n}\right)$. For, suppose $R$ is the complete matrix ring, $(G F(2))_{2}$, of all $2 \times 2$ matrices over $G F(2)$. It is easily verified that

$$
\begin{aligned}
x_{1} x_{2} & =x_{1}^{7} x_{2} & & \text { if } x_{1} \text { is invertible or idempotent } \\
& =x_{1} x_{2}^{7} & & \text { if } x_{2} \text { is invertible or idempotent } \\
& =\left(x_{1} x_{2}\right)^{2} & & \text { otherwise. }
\end{aligned}
$$

However, the commutator ideal of $(G F(2))_{2}$ is not even nil. In verifying (10), observe that (i) $x^{8}=x^{2}$ holds in $(G F(2))_{2}$; (ii) every matrix in $(G F(2))_{2}$ is invertible, or idempotent, or nilpotent; (iii) the product of any two nilpotent matrices in $(G F(2))_{2}$ is idempotent.

In conclusion, we wish to express our indebtedness and gratitude to the referee for his suggestions which resulted in shorter proofs and stronger results.

\section{REFERENCES}

1. I. N. Herstein, Theory of rings, Math. Lecture Notes, University of Chicago, Chicago, Ill., 1961.

2. N. Jacobson, Structure of rings, rev. ed., Amer. Math. Soc. Colloq. Publ., vol. 37, Amer. Math. Soc., Providence, R.I., 1964. MR 36 \#5158. 
3. N. H. McCoy, The theory of rings, Macmillan, New York; Collier-Macmillan, London, 1964. MR 32 \#5680.

4. M. S. Putcha and A. Yaqub, Semigroups satisfying permutation identities, Semigroup Forum 13 (1971), 68-73.

Department of Mathematics, University of California, Santa Barbara, California 93106

Current address (Putcha): Department of Mathematics, University of California, Berkeley, California 94720 